\title{
Acute Disseminated Encephalomyelitis Following Typhoid Fever: A Case Report
}

\author{
Adhikari $\mathbf{R}^{1}$, Tayal $\mathrm{A}^{2}$, Chhetri $\mathrm{PK}^{3}$, Pokhrel $\mathrm{B}^{4}$ \\ ${ }^{1}$ Resident, ${ }^{2,3}$ Associate Professor, Department of Radiodiagnosis, ${ }^{4}$ Resident, Department of \\ Neuromedicine, College of Medical Sciences and Teaching Hospital, Bharatpur, Nepal
}

\section{ABSTRACT \\ The involvement of central nervous system in children with typhoid fever is common. Acute disseminated encephalomyelitis is a rare immune mediated and demyelinating disease of the central nervous system that usually affects children. We report a 7-year-old child with typhoid fever who developed acute cerebellar syndrome due to acute disseminated encephalomyelitis.}

Keywords: acute disseminated encephalomyelitis; acute cerebellar syndrome; typhoid fever

\section{INTRODUCTION}

Typhoid fever still remains a major public health problem, with global annual incidence of about 21 million cases. ${ }^{1}$ Affected children typically present with fever, anorexia, headache, abdominal pains, diarrhoea, constipation and myalgia. However, central nervous system (CNS) involvement in typhoid fever is an important atypical presentation in childhood. Acute disseminated encephalomyelitis (ADEM) is a rare immune mediated and demyelinating disease of the brain and spinal cord and can be defined as scattered focal or multifocal inflammation of CNS. ${ }^{2}$ ADEM is a monophasic disease that usually develops following acute viral infection, vaccination or organ transplantation. ${ }^{3,4}$ This disease entity as a complication of typhoid fever is very rare and only few cases had been reported. ${ }^{5}$

Here we report a case of ADEM in a 7-year-old child with diagnosed typhoid fever, acute cerebellar syndrome and magnetic resonance imaging (MRI) evidence of ADEM.

Correspondence: Dr. Ridhi Adhikari

E-mail: ridhi.adhikari@gmail.com

\section{CASE REPORT}

A 7-year-old female child was admitted with acute onset difficulty in walking, and dysarthria. Symptoms got progressively worse over a period of 2 days. She has had a febrile illness four weeks prior to the present illness. That time a Widal test was positive with a Salmonella typhi $\mathrm{H}$ titer of more than 1:160 and she was diagnosed to have typhoid fever. On admission she had nystagmus, cerebellar and pyramidal tract signs and gait ataxia. The physical examination showed the patient's vital signs stable. Her past medical history was unremarkable. A lumbar puncture was performed. Cerebrospinal fluid (CSF) revealed predominant lymphocytosis, adenosine deaminase (ADA) of 2.4 $\mathrm{U} / \mathrm{L}$ (normal $<10 \mathrm{U} / \mathrm{L}$ ), a protein content of 33.8 $\mathrm{mg} / \mathrm{dL}$, and a glucose content of $55 \mathrm{mg} / \mathrm{dL}$. The CSF culture showed no growth of bacteria.

Computed tomography (CT) scan brain revealed ill-defined areas of hypodensity in the right 
Journal of College of Medical Sciences-Nepal, 2013, Vol-9, No-4, cerebellar hemisphere. MRI brain scan revealed hyperintense areas involving bilateral middle cerebellar peduncle, right cerebellar hemisphere, pons, right side of mid brain, bilateral periventricular and subcortical white matter in $\mathrm{T} 2$ weighted (T2WI) and Fluid-attenuated inversion recovery (FLAIR) showing open ring enhancement in T1 weighted post contrast images (Figure. 1, Figure. 2, and Figure. 3). Corpus callosum was intact.

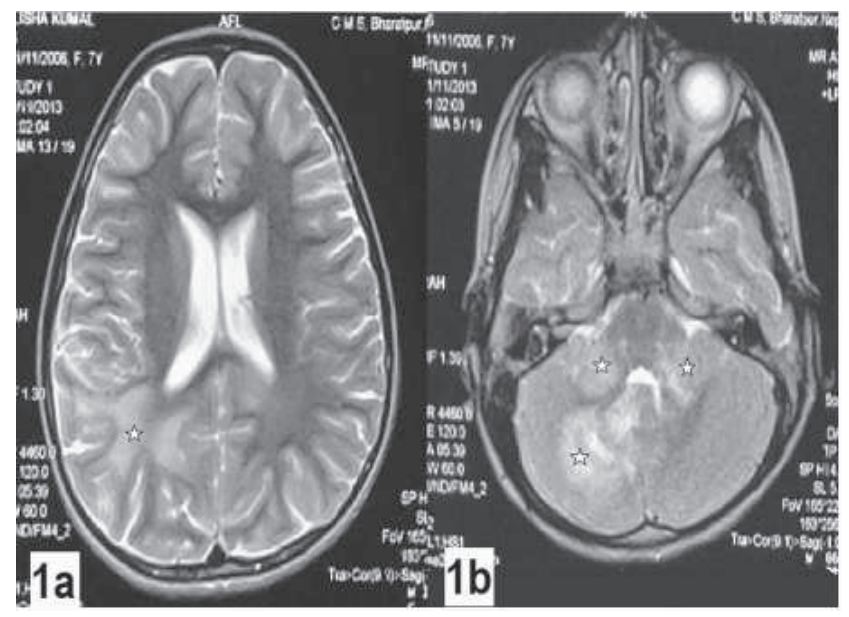

Fig 1a, 1b: Axial T2 weighted MRI brain scan revealed hyperintense areas involving bilateral middle cerebellar peduncle, right cerebellar hemisphere, right periventricular and subcortical white matter (stars)

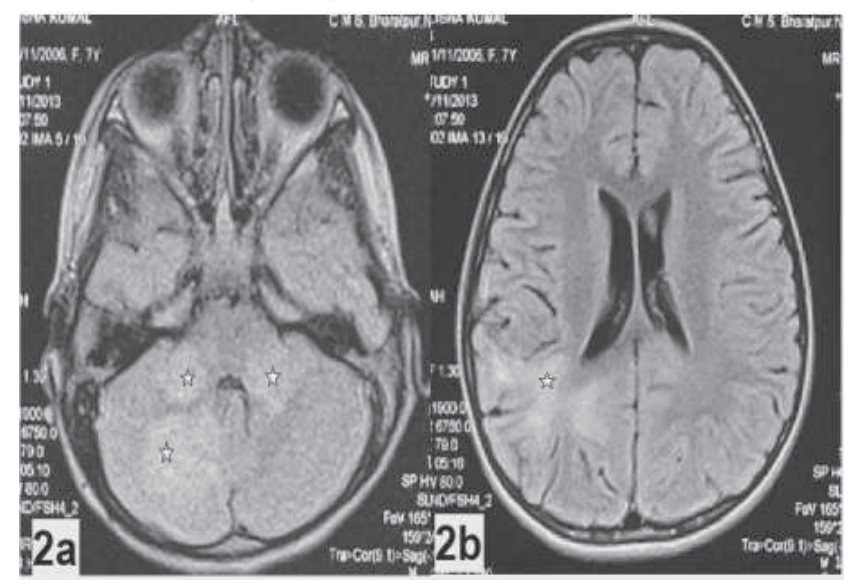

Fig 2a, 2b: Axial fluid-attenuated inversion recovery (FLAIR) MRI brain scan revealed hyperintense areas involving bilateral middle cerebellar peduncle, right cerebellar hemisphere, right periventricular and subcortical white matter (stars)

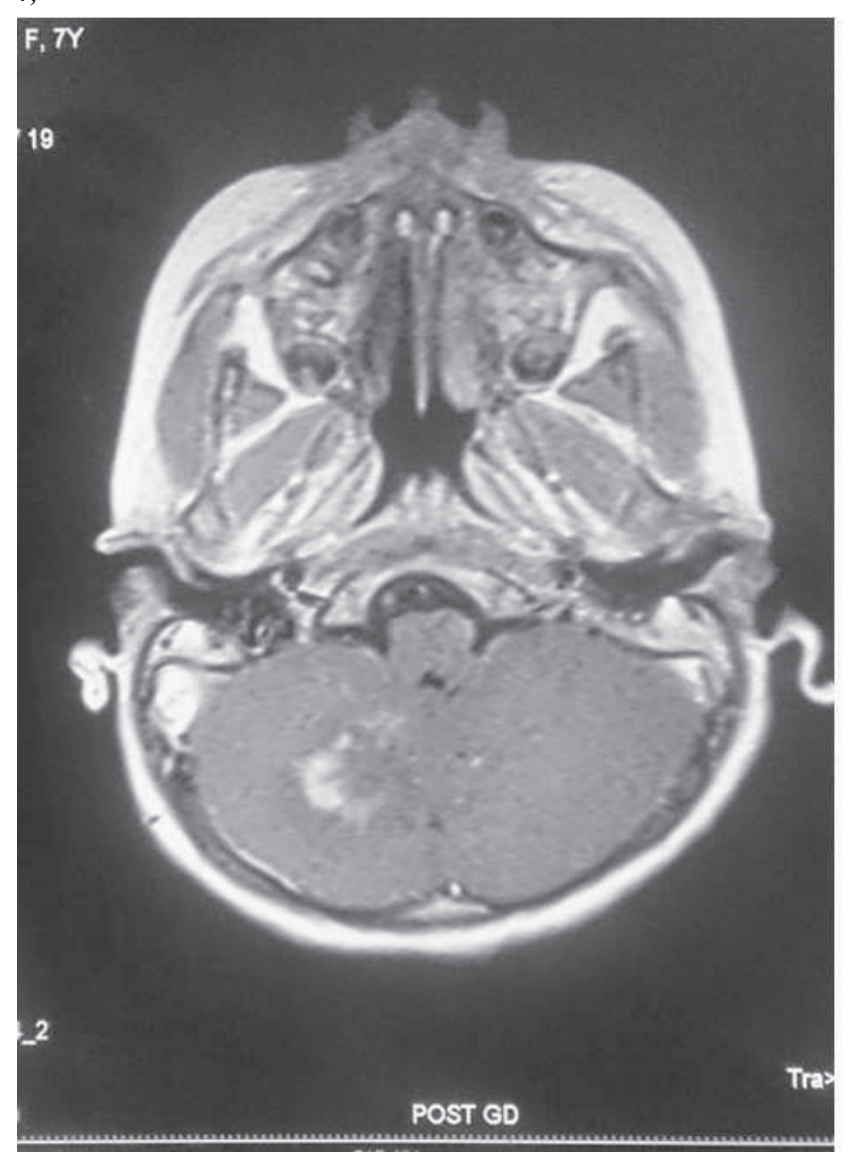

Fig 3: Post-contrast T1 weighted images showing open ring enhancement in the right cerebellum.

The clinical picture and the MRI scan findings were suggestive of acute disseminated encephalomyelitis (ADEM). She was started on high dose IV steroids. She made a dramatic improvement over the next few weeks and was able to walk well at the end of four weeks. On discharge, the patient was fully relieved from all previous symptoms.

\section{DISCUSSION}

ADEM is a rare acute autoimmune mediated disease characterized by a widespread demyelization that predominantly involves the white matter of the brain and spinal cord. ${ }^{2}$ It is an inflammatory process manifested by rapid onset of multifocal neurological impairment. ADEM usually develops following acute viral infection, vaccination or organ transplantation over days, weeks or months, punctuated by an acute worsening 
Acute Disseminated Encephalomyelitis Following Typhoid Fever: A Case Report

and is more frequent in children [3,4]. However, the present case report like few others ${ }^{5,6,7}$ showed that it can also be caused by bacterial infections such as typhoid fever.

The true incidence of ADEM is unknown. In one study, ADEM almost involves children with the incidence rate of $0.4 / 100000$ yearly in patients less than 20 years-old. ${ }^{8}$ The peak incidence of ADEM is among 5 to 8 years of age. ${ }^{9,10}$ Male to female ratio is the same.

Imaging is a valuable diagnostic tool. CT and MRI are worthwhile tools in establishing the diagnosis of ADEM. CT is often normal at first, but changes during 5-14 days later. CT changes include: multifocal lesions in subcortical zone of white matter with low attenuation. Of course MRI is the most extremely diagnostic tool because demyelinating lesions are better seen in MRI. Cerebellum and brain stem involvement are common. MRI characteristics include patchy lesions with severe increase of signals in conventional T2-WI and FLAIR. Although, white matter is the most common place of involvement, gray- matter especially basal ganglia, thalamus and brain stem can be involved. Corpus callosum is usually intact and its involvement is suggestive and has the characteristic of Multiple Sclerosis (MS). ${ }^{11,12,13}$ The other variables are cerebrospinal fluid (CSF) changes; often include increased CSF pressure, raised protein and lymphocytic pleocytosis. Glucose is usually in a normal range.

Multiple sclerosis (MS) is the main differential diagnosis of ADEM. In contrast to MS, ADEM is usually a monophasic disorder with favorable long term prognosis. Monophasic ADEM is more common in children whereas MS is more common in adults. It has been shown that the mean age was 7.1 years and 12 years in ADEM and MS patients respectively. ${ }^{14}$ However, history of typhoid fever, high load of lesions in MRI, early onset ataxia, deep cortical gray matter involvement and intact corpus callosum in our case highly suggested ADEM. This patient was definitely suffering from typhoid fever in the early stage, but subsequently it was complicated by the development of ADEM. After the MRI was scanned, the diagnosis of ADEM was made. Moreover, the diagnosis was also supported by the CSF findings (lymphocytic pleocytosis).

\section{CONCLUSION}

Typhoid fever is associated with several neurological complications including postinfectious demyelination as the underlying pathological process. It is usually reversible; however, it may be lethal if left untreated. Since clinical diagnosis of ADEM may not be clear initially and in the absence of brain MRI scan, pediatricians, particularly those who work in low resource countries like ours, should consider this diagnosis in children with typhoid fever associated with cerebellar dysfunction.

\section{REFERENCES}

1. World Health Organisation. Typhoid vaccines: World Health Organisation position paper. Weekly Epidemiol Rec 2008; 83:49-60.

2. Garg RK. Acute disseminated encephalomyelitis. Postgrad Med J 2003;79:117

3. Menge T, Hemmer B, Nessler S, et al. Acute Disseminated Encephalomyelitis an update. Arch Neurol 2005;62:1673-80.

4. Wender M. Acute disseminated encephalomyelitis (ADEM). J Neuroimmunol 2011;231:92-9.

5. Adegoke SA, Ayoola OO, Oseni SBA. Acute disseminated encephalomyelitis in two 
Journal of College of Medical Sciences-Nepal, 2013, Vol-9, No-4,

Nigerian children with typhoid fever. Niger J Paed 2012;39:140-3.

6. Lakhotia M, Gehlot RS, Jain P, et al. Neurological manifestations of enteric fever. $\mathbf{J}$ Indian Acad Clin Med 2003;4:196-9.

7. Arii J, Tanabe Y, Miyake M, et al. Clinical and pathologic characteristics of nontyphoidal Salmonella Encephalopathy. Neurology 2002;58:1641-54.

8. Leake JA, Albani S, Kao AS, et al. Acute disseminated encephalomyelitis in childhood: epidemiologic, clinical and laboratory features. Pediatr Infect Dis J 2004;23:756-64.

9. Sabayan B,Zolghadrasli A.Vasculitis and rheumatologic diseases may play role in the pathogenesis of acute disseminated encephalomyelitis ADEM). Med Hypotheses 2007;69:322-4.
10. Marchioni E, Tavazzi E, Minoli L, et al. Acute disseminated encephalomyelitis. Neurol Sci 2008;29:S286-8.

11. Hung PC, Wang HS, Chou ML, et al. Acute disseminated encephalomyelitis in children: a single institution experience of 28 patients. Neuropediatrics 2012;43:64-71.

12. Monden Y, Yamagata T, Kuroiwa Y, et al. A case of ADEM with atypical MRI findings of a centrally-located long spinal cord lesion. Brain Dev 2012;34:380-3.

13. Alper G. Acute disseminated encephalomyelitis. J Child Neurol 2012;27:1408-25.

14. Mikaeloff Y, Suissa S, Vallee L, et al. First episode of acute CNS inflammatory demyelination in childhood; Prognostic factors for multiple sclerosis \& disability. J Pediatr 2004;144:246-52. 